\title{
Some consequences in weak processes of three-generation mixing in the leptonic sector
}

\author{
O. L. G. Peres and V. Pleitez \\ Instituto de Física Teórica, Universidade Estadual Paulista, Rua Pamplona 145, 01405-900 São Paulo, SP, Brazil \\ R. Zukanovich Funchal \\ Instituto de Física da Universidade de São Paulo, 01498-970 C.P. 20516 São Paulo, SP, Brazil
}

(Received 11 October 1993)

\begin{abstract}
We investigate the sensitivity of some weak processes to a Cabibbo-Kobayashi-Maskawa mixing in the leptonic sector. Values for mixing angles and masses compatible with several experimental accelerator data were found. We discuss in this context neutrino oscillations and cosmological and astrophysical consequences as well.
\end{abstract}

PACS number(s): 14.60.Pq, 13.35.-r

\section{INTRODUCTION}

The physics of the $\tau$ lepton will provide in the near future evidence concerning the question if this lepton, with its neutrino partner, is a sequential lepton or not. Although the existing data indicate a positive answer to that question, the structure of the weak interaction of the third lepton family deserves more detailed experimental and theoretical studies. As was stressed in Ref. [1], all experiments are internally consistent with the standard model. Notwithstanding, it is well known that the accuracy of the $\tau$ data is still poor and it should be possible that new physics will come up when the proposed $\tau$-charm factories give new and more accurate data about $\tau$ decays and properties [2].

One important issue concerns lepton universality. The $e-\mu$ universality is well established in $\pi$ decays given a ratio [3]

$$
G_{e} / G_{\mu}=0.9985 \pm 0.0015
$$

On the other hand, the current data on $\tau-\mu$ universality have not the same precision and one still can speculate on a possible departure from $\tau-\mu$ universality. is

A quantity which parametrizes the $\tau-\mu$ universality

$$
\left(\frac{G_{\tau}}{G_{\mu}}\right)^{2}=\left(\frac{\tau_{\mu}}{\tau_{\tau}}\right)\left(\frac{m_{\mu}}{m_{\tau}}\right)^{5} \frac{B^{\tau e}}{B^{\mu e}}
$$

where $G_{\tau}$ and $G_{\mu}$ are, respectively, the coupling constants of the $\tau$ and $\mu$ to the charged weak current, respectively. We will use the notation $B^{a b}=B(a \rightarrow b+X)$ where $X$ are appropriate particles. A similar notation is used for the partial width $\Gamma^{a b}$.

The new values for the $\tau$ mass $m_{\tau}=1776.9_{-0.5}^{+0.4} \pm 0.2$ $\mathrm{MeV}$ [4] or $m_{\tau}=1771.3 \pm 2.4 \pm 1.4 \mathrm{MeV}$ [5] imply that a possible deviation from $\tau-\mu$ universality is reduced from $2.5 \sigma$ to $1.7 \sigma$. Explicitly with the BES data one has

$$
\left(\frac{G_{\tau}}{G_{\mu}}\right)^{2}=0.960 \pm 0.024
$$

Two other experimental quantities which we will use later on are [6]

$$
\frac{B^{\tau \mu}}{B^{\tau e}}=0.98 \pm 0.02
$$

and

$$
\frac{B^{\pi e}}{B^{\pi \mu}}=(1.218 \pm 0.014) \times 10^{-4}
$$

More recent data give for the ratio in (1.5) [7]

$$
1.2265 \pm 0.0034(\text { stat }) \pm 0.0044 \text { (syst) } \times 10^{-4}
$$

however we will use, for consistence, the world average of Ref. [6] for all quantities.

In this paper we will consider that if neutrinos are massive in the standard electroweak model [8], a mixing similar to the Cabibbo-Kobayashi-Maskawa (CKM) one in the quark sector [9], may occur with three lepton generations. We show in this paper that such a mixing it is not ruled out by current data and can be used to explain a possible deviation from $\tau-\mu$ universality.

We must nevertheless stress that the analysis we will put forward in this work is valid even if $\tau-\mu$ universality is confirmed. Only the numerical values that we give will change.

The introduction of a mixing among three generations makes room for a third massive neutrino with a mass, as we will see, beyond the current limit of what is consider, without mixing, the " $\tau$ neutrino" $[5,10]$.

The existence of neutral heavy leptons has been discussed in the literature since about 20 years ago [11]. Usually, the heavy neutrino is assumed to mix with the electron and muon families with either $V-A$ or $V+A$ charged and neutral currents. The heavy neutrino could be a higher generation neutrino, associated with a yet unobserved heavy charged lepton, a right-handed neutrino, 
etc. [12]. Another possibility usually considered is that in addition to the three left-handed lepton doublets one includes $k$ neutrino singlets $\chi_{j}, j=1, \ldots, k[13,14]$. This led to neutrino mixing without affecting the $Z^{0}$ width.

Here we will suppose that only 3 right-handed singlets have been added and that there is no Majorana mass terms among the right-handed singlets. This is the simplest extension of the standard model which includes massive Dirac neutrinos. The only supposition we will make is that

$$
0 \lesssim m_{\nu_{1}} \approx m_{\nu_{2}} \ll m_{\nu_{3}}
$$

This will be assumed not only for the sake of simplicity but because it is an interesting possibility. The two light neutrinos could be detected in oscillation experiments [15]. We will not make any further assumptions concerning the mixing matrix elements nor the $m_{\nu_{3}}$ value but leave them to be constrained by several experimental data. This means that we will fit in our analysis, at the same time, two mixing angles and a mass.

We stress that it is important to specify the model one is considering when comparing with experimental data. A purely model-independent approach cannot be definitive.

The outline of this work is as follows. In Sec. II we consider the effect of mixing in the partial width for the muon decay, leptonic $\tau$ decays and pion decays. These are the quantities which are calculated theoretically. In Sec. III we compare our theoretical results with experimental data (within $1 \sigma$ ) for $\tau$ and pion decays deriving from this comparison regions of permitted mixing angles and mass. In Sec. IV A we take into account the constraints coming from the $Z^{0}$ invisible partial width. In Sec. IV B we discuss the current limit of " $m_{\nu_{\tau}}$ " on the light of the mixing angles obtained in Sec III, showing that it is possible to go beyond the current limit of 31 $\mathrm{MeV}$ without contradicting experimental results. Neutrino oscillations are consider in Sec. V A; cosmological and astrophysical constraints are discussed in Sec. V B and Sec. VC, respectively. Finally, the last section is devoted to our conclusions.

\section{THREE GENERATIONS MIXING IN THE LEPTONIC SECTOR}

In this paper we will consider only Dirac neutrinos. The general effects of the Cabibbo-KobayashiMaskawa mixing in the leptonic sector were considered in Refs. [16-18]. In particular the effects of such a mixing for the case of leptonic decays of the $\mu$ were explicitly considered in Ref. [19], and in Ref. [20] for the $\tau$ lepton case.

In fact, it has been shown by Shrock [17] that if neutrinos have nonzero masses the semileptonic decays $h^{\prime} \rightarrow h+l+\nu_{l}$ consist of an incoherent sum of the separate modes $h^{\prime} \rightarrow h+l+\nu_{i}$, where $\nu_{l}=\nu_{e}, \nu_{\mu}, \nu_{\tau}$ are weak eigenstates and $\nu_{i}, i=1,2,3$ are the mass eigenstates allowed by phase space. These states are related by $\nu_{l}=\sum_{i} V_{l i} \nu_{i}, V$ being the unitary leptonic CKM matrix. Explicitly, this mixing matrix is defined for the three generations case as

$$
\left(\begin{array}{c}
\nu_{e} \\
\nu_{\mu} \\
\nu_{\tau}
\end{array}\right)=\left(\begin{array}{lll}
V_{e 1} & V_{e 2} & V_{e 3} \\
V_{\mu 1} & V_{\mu 2} & V_{\mu 3} \\
V_{\tau 1} & V_{\tau 2} & V_{\tau 3}
\end{array}\right)\left(\begin{array}{c}
\nu_{1} \\
\nu_{2} \\
\nu_{3}
\end{array}\right)
$$

It is well known that there are several parametrizations of the CKM matrix. Here we will use the Maiani one $[6,21]$. This form has the feature that when two of such angles vanish the mixing matrix reduces to the usual Cabibbo-like mixing matrix of two generations. Using the usual notation $c_{i j}=\cos \theta_{i j}, s_{i j}=\sin \theta_{i j}$, with $i$ and $j$ being generation labels $i, j=1,2,3$, setting the phase $\delta_{13}=0$ for the sake of simplicity and making the substitution $c_{12} \rightarrow c_{\theta}, c_{13} \rightarrow c_{\beta}$ and $c_{23} \rightarrow c_{\gamma}$ we have

$$
\left(\begin{array}{ccl}
c_{\theta} c_{\beta} & s_{\theta} c_{\beta} & s_{\beta} \\
-s_{\theta} c_{\gamma}-c_{\theta} s_{\gamma} s_{\beta} & c_{\theta} c_{\gamma}-s_{\theta} s_{\gamma} s_{\beta} & s_{\gamma} c_{\beta} \\
s_{\theta} s_{\gamma}-c_{\theta} c_{\gamma} s_{\beta} & -c_{\theta} s_{\gamma}-s_{\theta} c_{\gamma} s_{\beta} & c_{\gamma} c_{\beta}
\end{array}\right) .
$$

Assuming that only one of the neutrinos, say $\nu_{3}$, is sufficiently massive we can write for the decay probability of a charged lepton $l^{\prime}$ into the charged lepton $l$ and neutrinos $\nu_{i} \bar{\nu}_{j}$ the expression [20]

$$
\begin{aligned}
\Gamma\left(l^{\prime} \rightarrow l \bar{\nu}_{l} \nu_{l^{\prime}}\right)= & \frac{G^{2} m_{l^{\prime}}^{5}}{192 \pi^{3}}\left\{\left(\left|V_{l^{\prime} 1}\right|^{2}+\left|V_{l^{\prime} 2}\right|^{2}\right)\left(\left|V_{l 1}\right|^{2}+\left|V_{l 2}\right|^{2}\right) \Gamma_{00}^{l^{\prime} l}+\left(\left|V_{l 3}\right|^{2}\left[\left|V_{l^{\prime} 1}\right|^{2}+\left|V_{l^{\prime} 2}\right|^{2}\right]+\left|V_{l^{\prime} 3}\right|^{2}\left[\left|V_{l 1}\right|^{2}+\left|V_{l 2}\right|^{2}\right]\right) \Gamma_{03}^{l^{\prime} l}\right. \\
& \left.+\left|V_{l^{\prime} 3}\right|^{2}\left|V_{l 3}\right|^{2} \Gamma_{33}^{l^{\prime \prime} l}\right\}
\end{aligned}
$$

with $l^{\prime}=\mu, \tau$ and $l=e, \mu$ for the $\tau$ decay and $l=e$ for the muon decay. Notice that $G^{2}$, although it is still defined as $G^{2} / \sqrt{2}=g^{2} / 8 m_{W}^{2}$ in Eq. (2.3) is not equal to the muon decay constant $G_{\mu}$. We shall return to this point later. In (2.3) we have defined the integrals

$$
\Gamma_{00}^{l^{\prime} l}=2 \int_{x_{m}}^{x_{M}}\left(x^{2}-B\right)^{\frac{1}{2}}[x(3 k-2 x)-B] d x
$$




$$
\begin{gathered}
\Gamma_{03}^{l^{\prime} l}=2 \int_{x_{m}}^{x_{M}}\left(x^{2}-B\right)^{\frac{1}{2}} \frac{\left(k-\delta_{3 l^{\prime}}^{2}-x\right)}{(k-x)^{3}}\left[\left(k-\delta_{3 l^{\prime}}^{2}-x\right)^{2} x(k-x)\right. \\
\left.+\left[(k-x)^{2}+\delta_{3 l^{\prime}}^{2}(k-x)-2 \delta_{3 l^{\prime}}^{4}\right]\left(2 k x-x^{2}-B\right)\right] d x, \\
\Gamma_{33}^{l^{\prime} l}=2 \int_{x_{m}}^{x_{M}} \frac{\left(x^{2}-B\right)^{\frac{1}{2}}}{(k-x)^{\frac{3}{2}}}\left(k-x-4 \delta_{3 l^{\prime}}^{2}\right)^{\frac{1}{2}}\left[\left(k-x-4 \delta_{3 l^{\prime}}^{2}\right) x(k-x)+\left(k-x+2 \delta_{3 l^{\prime}}^{2}\right)\left(2 k x-x^{2}-B\right)\right] d x,
\end{gathered}
$$

with

$$
\begin{gathered}
k=1+\delta_{l l^{\prime}}^{2}, \quad B=4(k-1), \quad \delta_{3 l^{\prime}}=\frac{m_{\nu_{3}}}{m_{l^{\prime}}}, \quad \delta_{l l^{\prime}}=\frac{m_{l}}{m_{l^{\prime}}} \\
x_{m}=2 \delta_{l l^{\prime}}, \quad x_{M}=k-\frac{\left(m_{\nu_{i}}+m_{\nu_{j}}\right)^{2}}{m_{l^{\prime}}^{2}} .
\end{gathered}
$$

$\Gamma_{00}^{l^{\prime} l}, \Gamma_{03}^{l^{\prime} l}$, and $\Gamma_{33}^{l^{\prime} l}$ are the contributions for the $l^{\prime} \rightarrow l \bar{\nu}_{i} \nu_{j}$ decays from two massless, one massive, and two massive neutrinos, respectively.

Using explicitly the parametrization in (2.2) we obtain

$$
\begin{gathered}
\Gamma\left(\mu \rightarrow e \nu_{\mu} \bar{\nu}_{e}\right)=\frac{G^{2} m_{\mu}^{5}}{192 \pi^{3}}\left[\left(s_{\beta}^{2} s_{\gamma}^{2}+c_{\gamma}^{2}\right) c_{\beta}^{2} \Gamma_{00}^{\mu e}+\left(s_{\beta}^{4} s_{\gamma}^{2}+s_{\beta}^{2} c_{\gamma}^{2}+c_{\beta}^{4} s_{\gamma}^{2}\right) \Gamma_{03}^{\mu e}+s_{\gamma}^{2} c_{\beta}^{2} s_{\beta}^{2} \Gamma_{33}^{\mu e}\right] \\
\Gamma\left(\tau \rightarrow e \nu_{\tau} \bar{\nu}_{e}\right)=\frac{G^{2} m_{\tau}^{5}}{192 \pi^{3}}\left[\left(s_{\beta}^{2} c_{\gamma}^{2}+s_{\gamma}^{2}\right) c_{\beta}^{2} \Gamma_{00}^{\tau e}+\left(s_{\beta}^{4} c_{\gamma}^{2}+s_{\beta}^{2} s_{\gamma}^{2}+c_{\beta}^{4} c_{\gamma}^{2}\right) \Gamma_{03}^{\tau e}+c_{\gamma}^{2} c_{\beta}^{2} s_{\beta}^{2} \Gamma_{33}^{\tau e}\right] \\
\Gamma\left(\tau \rightarrow \mu \nu_{\tau} \bar{\nu}_{\mu}\right)=\frac{G^{2} m_{\tau}^{5}}{192 \pi^{3}}\left(\left(s_{\beta}^{2} c_{\gamma}^{2}+s_{\gamma}^{2}\right)\left(s_{\beta}^{2} s_{\gamma}^{2}+c_{\gamma}^{2}\right) \Gamma_{00}^{\tau \mu}\right. \\
\left.+\left(s_{\gamma}^{2}\left(s_{\beta}^{2} c_{\gamma}^{2}+s_{\gamma}^{2}\right)+c_{\gamma}^{2}\left(s_{\beta}^{2} s_{\gamma}^{2}+c_{\gamma}^{2}\right)\right) c_{\beta}^{2} \Gamma_{03}^{\tau \mu}+c_{\gamma}^{2} s_{\gamma}^{2} c_{\beta}^{4} \Gamma_{33}^{\tau \mu}\right]
\end{gathered}
$$

Notice that Eqs. (2.9)-(2.11) depend only on the angles $\beta$ and $\gamma$. We see from Eq. (2.9) that in fact

$$
G_{\mu}^{2}=G^{2} \times \text { factor, }
$$

where the factor will depend on the kinematical region allowed for a particular decay, so that the constant $G^{2}$ appearing in other decays is that given by Eq. (2.12). For instance, if $\nu_{3}$ is kinematically forbidden in the muon decay, from (2.9) we obtain

$$
\begin{aligned}
G_{\mu}^{2} & =G^{2}\left(1-\left|V_{\mu 3}\right|^{2}\right)\left(1-\left|V_{e 3}\right|^{2}\right) \\
& =G^{2}\left(s_{\beta}^{2} s_{\gamma}^{2}+c_{\gamma}^{2}\right) c_{\beta}^{2} .
\end{aligned}
$$

Hence, we have defined $G_{\mu}$ in such a way that it coincides with the usual value.

We will write Eqs. (2.9), (2.10), and (2.11), respectively, in the form

$$
\Gamma\left(\mu \rightarrow e \nu_{\mu} \bar{\nu}_{e}\right)=\frac{G^{2} m_{\mu}^{5}}{192 \pi^{3}} f^{\mu e}\left(\beta, \gamma, \delta_{e \mu}, \delta_{3 \mu}\right),
$$

for the partial rate of the muon decay into electron and

$$
\begin{gathered}
\Gamma\left(\tau \rightarrow e \nu_{\tau} \bar{\nu}_{e}\right)=\frac{G^{2} m_{\tau}^{5}}{192 \pi^{3}} f^{\tau e}\left(\beta, \gamma, \delta_{e \tau}, \delta_{3 \tau}\right), \\
\Gamma\left(\tau \rightarrow \mu \nu_{\tau} \bar{\nu}_{\mu}\right)=\frac{G^{2} m_{\tau}^{5}}{192 \pi^{3}} f^{\tau \mu}\left(\beta, \gamma, \delta_{\mu \tau}, \delta_{3 \tau}\right),
\end{gathered}
$$

for the decay of $\tau$ into electron and muon, respectively. Using Eqs. (2.14)-(2.16) we obtain

$$
\left(\frac{G_{\tau}}{G_{\mu}}\right)^{2}=\frac{f^{\tau e}\left(\beta, \gamma, \delta_{e \tau}, \delta_{3 \tau}\right)}{f^{\mu e}\left(\beta, \gamma, \delta_{e \mu}, \delta_{3 \mu}\right)}
$$

and

$$
\frac{B_{\mu}^{\tau}}{B_{e}^{\tau}}=\frac{f^{\tau \mu}\left(\beta, \gamma, \delta_{\mu \tau}, \delta_{3 \tau}\right)}{f^{\tau e}\left(\beta, \gamma, \delta_{e \tau}, \delta_{3 \tau}\right)}=\frac{\Gamma_{\mu}^{\tau}}{\Gamma_{e}^{\tau}} .
$$

The left-hand sides of (2.17) and (2.18) have the value showed in (1.3) and (1.4), respectively.

We will also consider $\pi \rightarrow l+\nu_{l}, l=e, \mu$ decays. The partial width with a massive neutrino is [17]

$$
\begin{aligned}
\Gamma_{3}^{\pi l}= & \frac{G^{2} f_{\pi}^{2} V_{\mathrm{KM}}^{2} m_{\pi}^{3}}{8 \pi}\left[\delta_{l \pi}^{2}+\delta_{3 \pi}^{2}\right. \\
& \left.-\left(\delta_{l \pi}^{2}-\delta_{3 \pi}^{2}\right)^{2}\right] \lambda^{\frac{1}{2}}\left(1, \delta_{l \pi}^{2}, \delta_{3 \pi}^{2}\right),
\end{aligned}
$$

where $\lambda$ is the triangular function, $\delta_{l \pi}=m_{l} / m_{\pi}$ and $V_{\mathrm{KM}}^{2}$ is the appropriate Cabibbo-Kobayashi-Maskawa matrix elements of the quark sector. In particular we have

$$
\begin{aligned}
\Gamma\left(\pi \rightarrow \mu \nu_{\mu}\right) & =\left(\left|V_{\mu 1}\right|^{2}+\left|V_{\mu 2}\right|^{2}\right) \Gamma_{0}^{\pi \mu}+\left|V_{\mu 3}\right|^{2} \Gamma_{3}^{\pi \mu} \\
& =\left(s_{\gamma}^{2} s_{\beta}^{2}+c_{\gamma}^{2}\right) \Gamma_{0}^{\pi \mu}+s_{\gamma}^{2} c_{\beta}^{2} \Gamma_{3}^{\pi \mu}, \\
\Gamma\left(\pi \rightarrow e \nu_{e}\right) & =\left(\left|V_{e 1}\right|^{2}+\left|V_{e 2}\right|^{2}\right) \Gamma_{0}^{\pi e}+\left|V_{e 3}\right|^{2} \Gamma_{3}^{\pi e} \\
& =c_{\beta}^{2} \Gamma_{0}^{\pi e}+s_{\beta}^{2} \Gamma_{3}^{\pi e} .
\end{aligned}
$$


From (2.20) and (2.21) we obtain

$$
\frac{\Gamma^{\pi e}}{\Gamma^{\pi \mu}}=\frac{c_{\beta}^{2} \Gamma_{0}^{\pi e}+s_{\beta}^{2} \Gamma_{3}^{\pi e}}{\left(s_{\gamma}^{2} s_{\beta}^{2}+c_{\gamma}^{2}\right) \Gamma_{0}^{\pi \mu}+s_{\gamma}^{2} c_{\beta}^{2} \Gamma_{3}^{\pi \mu}}=\frac{B^{\pi e}}{B^{\pi \mu}} .
$$

In the next section we will compare Eqs. (2.17), (2.18), and (2.22) with (1.3)-(1.5) and other experimental data.

\section{EXPERIMENTAL CONSTRAINTS ON MIXING ANGLES AND NEUTRINO MASS}

The effects of a leptonic mixing in the decays of pseudoscalars mesons was studied by Shrock [17] and $\mathrm{Ng}$ [18]. Since then, several experiments were performed searching the effect of extra peaks due to massive neutrinos in the spectrum of the positron in $\pi^{+} \rightarrow e^{+} \nu(\gamma)$ [22]. The measurement of this branching ratio confirm the hypothesis of the $e-\mu$ universality at the $0.2 \%$ level [3]. The theoretical uncertainty arises from structure-dependent loop effects, they could be important in the $\pi \rightarrow e \nu_{e} \gamma$ decay, but are less than $0.1 \%$ [3].

Here we will point out some considerations about these experiments. In most of them when fitting experimental data with theoretical calculations it have been assumed that the heavy neutrino couples mainly with the electron or muon. Hence, according to this what they are comparing with experimental data is [23]

$$
\frac{\Gamma\left(\pi \rightarrow l \nu_{3}\right)}{\Gamma\left(\pi \rightarrow l \nu_{l}\right)} \propto\left|V_{l 3}\right|^{2}
$$

where $\nu_{l}$ is the conventional massless neutrino, $l=e, \mu$ and the factor of proportionality is the kinematical factor including phase space.

The ratio

$$
\frac{\Gamma\left(\pi \rightarrow e \nu_{3}\right)}{\Gamma\left(\pi \rightarrow \mu \nu_{l}\right)}
$$

has also been considered by Bryman et al. [22], which depends on both $V_{e 3}, V_{\mu 3}$. However, the value of $V_{\mu 3}$ was taken from the work by Abela et al. [22]. In fact, most of the experimental data were fitted assuming a heavy subdominant coupled (HSC) neutrino, i.e., a heavy neutrino with $V_{l i} \ll 1[24]$.

In our analysis we will not make any assumption with respect to the matrix elements of the mixing matrix and when we have considered a kinematically allowed heavy neutrino in pion decays it is necessary to use (2.20) and (2.21) in which $V_{e 3, \mu 3}$ appear (after using the unitarity condition on the matrix $V$ ).

We have done several plots of Eqs. (2.17), (2.18), and (2.22) as a function of the angles $\beta$ and $\gamma$ for several values of $m_{\nu_{3}}$ using the experimental value (within $1 \sigma$ ) given by (1.3), (1.4), and (1.5). The values of the other parameters are those of the Ref. [6].

Let us consider the following intervals.

(i) $G_{\tau} / G_{\mu}>1$ independently of the mixing angles. For the experimental data we have used, this region corresponds to $m_{\nu_{3}}<50 \mathrm{MeV}$.

(ii) $G_{\tau} / G_{\mu} \leq 1$, but there is no intersection with (1.3).

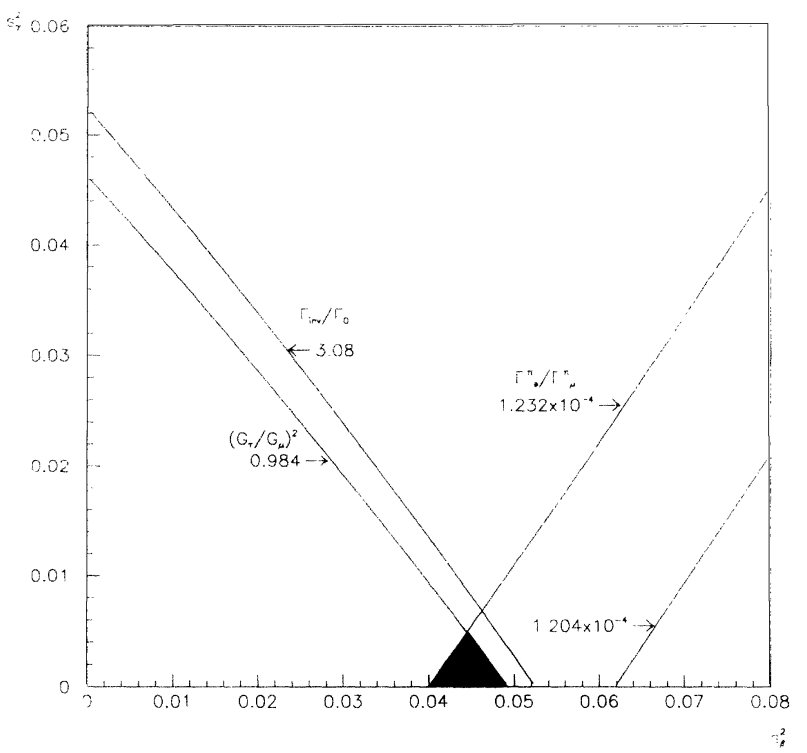

FIG. 1. Allowed region (within $1 \sigma$ ) in the plane $s_{\beta}^{2} \times s_{\gamma}^{2}$ for the ratios $G_{\tau} / G_{\mu}$ [Eq. (1.3)], $B^{\tau \mu} / B^{\tau e}$ [Eq. (1.4)], and $B^{\pi e} / B^{\pi \mu}$ [Eq. (1.5)] for $m_{\nu_{3}}=165 \mathrm{MeV}$. The region for $B^{\tau \mu} / B^{\tau e}$ is larger than the area shown in the picture. We will also show the contour plot of [Eq. (4.1)]. The black area is the allowed region combining all ratios.

This corresponds to the mass interval $50 \mathrm{MeV}<m_{\nu_{3}}<$ $84 \mathrm{MeV}$.

(iii) $G_{\tau} / G_{\mu} \leq 1$, compatible with (1.3) and (1.4) but not compatible with (1.5). In this case we have $84 \mathrm{MeV} \leq$ $m_{\nu_{3}}<155 \mathrm{MeV}$ and $m_{\nu_{3}}>800 \mathrm{MeV}$.

(iv) $G_{\tau} / G_{\mu} \leq 1$, compatible with all experimental data in the $m_{\nu_{3}}$ interval: $155 \mathrm{MeV} \leq m_{\nu_{3}} \leq 800 \mathrm{MeV}$.

In Fig. 1 we show contour plot of $(2.17),(2.18)$, and (2.22). The allowed values for the mixing angles we obtain, using $m_{\nu_{3}}=165 \mathrm{MeV}$, are

$$
11.54^{\circ}<\beta<12.82^{\circ}, \quad \gamma<4.05^{\circ} .
$$

We have seen that, to be in agreement with the processes represented by the quantities in Eqs. (2.17), (2.18), and (2.22) the heavier neutrino could have a mass in the interval:

$$
155 \mathrm{MeV} \lesssim m_{\nu_{3}} \lesssim 800 \mathrm{MeV}
$$

\section{CONSTRAINTS COMING FROM $Z$-INVISIBLE WIDTH AND END POINT OF $\tau$ DECAY INTO FIVE PIONS}

We have seen in the last section that the mass region allowed by the processes (1.3), (1.4), and (1.5) is given in (3.4). However, it is mandatory to consider the $Z^{0}$ invisible width and also the end point of the spectrum of the $\tau \rightarrow 5 \pi \nu_{\tau}$ decay which provide the current upper limit on the " $\tau$ neutrino" mass. Hence, we will continue our analysis in order to constrain even more the allowed values for the neutrino mass and mixing angles. 


\section{A. $Z^{0}$ invisible width}

Let us consider the constraints coming from the $Z^{0}$ invisible width measured by the experiments at the CERN $e^{+} e^{-}$collider LEP: $\Gamma^{\text {inv }}=502 \pm 9 \mathrm{MeV}$ [6]. For two massless and one massive neutrinos we have [25]

$$
\frac{\Gamma^{\mathrm{inv}}}{\Gamma^{0}}=\left[2+\sqrt{1-4 \mu_{F}}\left(1-\mu_{F}\right)\right] \frac{1}{\left[\left(s_{\beta}^{2} s_{\gamma}^{2}+c_{\gamma}^{2}\right) c_{\beta}^{2}\right]^{\frac{1}{2}}}
$$

where

$$
\begin{gathered}
\Gamma^{0}=\frac{M_{Z}^{3} G_{\mu}}{12 \sqrt{2} \pi}, \quad \mu_{F}=m_{\nu_{3}}^{2} / M_{Z}^{2} \\
M_{Z}=91.173 \pm 0.020 \mathrm{GeV}
\end{gathered}
$$

and

$$
G_{\mu}=1.16639 \times 10^{-5} \mathrm{GeV}^{-2},
$$

and we have used (2.13).

The contour plot of Eq. (4.1) for $m_{\nu_{3}}=165 \mathrm{MeV}$ appears also in Fig. 1. For masses above $225 \mathrm{MeV}$ there is no compatibility region among $Z^{0}$ data and the other experimental ratios we have considered in Sec. III. Hence, instead of (3.4) we have now

$$
155 \mathrm{MeV} \lesssim m_{\nu_{3}} \lesssim 225 \mathrm{MeV}
$$

For now on in our analysis we will use a typical value of $m_{\nu_{3}}=165 \mathrm{MeV}$ unless otherwise stated.

\section{B. End point of $\tau \rightarrow 5 \pi \nu_{\tau}$ in the mixing scenario}

The current upper limits on neutrino masses are

$$
\begin{array}{cc}
\text { " } m_{\nu_{e}} "<7.3 \mathrm{eV} & {[6],} \\
\text { " } m_{\nu_{\mu}} "<0.27 \mathrm{MeV} & {[6]} \\
\text { " } m_{\nu_{\tau}} "<31 \mathrm{MeV} & {[5,10] .}
\end{array}
$$

However these values were obtained in experiments which tried to observe the effects of neutrino masses without mixing in the leptonic sector.

The current experimental upper bound on " $m_{\nu_{r}}$ " given by ARGUS $[5,10]$ comes from the study of the end point of the hadronic invariant mass distribution in the decay $\tau \rightarrow 5 \pi \nu_{\tau}$. Their analysis is only valid when there is no mixing in the leptonic sector, otherwise the massive neutrino will not manifest itself by a shift on the end point but by a shoulder in the distribution. The end point in both situations will be the same.

For the sake of simplicity we shall consider here only the $\tau$ neutrino case but a similar procedure could be applied to the other $e$ and $\mu$ neutrinos. Assuming that $\nu_{\tau}$ in the decay $\tau \rightarrow n \pi \nu_{\tau}$ is a particle with definite mass it was obtained [26]

$$
\frac{d \Gamma}{d q^{2}}=\frac{G^{2} V_{\mathrm{KM}}^{2}}{8 \pi m_{\tau}^{3}} w\left(q^{2}, m_{\tau}^{2}, m_{\nu_{\tau}}^{2}\right) \lambda^{\frac{1}{2}}\left(m_{\tau}^{2}, q^{2}, m_{\nu_{\tau}}^{2}\right) h\left(q^{2}\right)
$$

where

$$
\begin{aligned}
w\left(q^{2}, m_{\tau}^{2}, m_{\nu_{\tau}}^{2}\right)= & \left(m_{\tau}^{2}-q^{2}\right)\left(m_{\tau}^{2}+2 q^{2}\right) \\
& -m_{\nu_{\tau}}^{2}\left(2 m_{\tau}^{2}-q^{2}-m_{\nu_{\tau}}^{2}\right)
\end{aligned}
$$

and

$$
\begin{aligned}
\lambda^{\frac{1}{2}}\left(m_{\tau}^{2}, q^{2}, m_{\nu_{\tau}}^{2}\right)= & {\left[m_{\tau}^{2}-\left[\left(q^{2}\right)^{\frac{1}{2}}+m_{\nu_{\tau}}\right]^{2}\right\} } \\
& \left.\times\left\{m_{\tau}^{2}-\left[\left(q^{2}\right)^{\frac{1}{2}}-m_{\nu_{\tau}}\right]^{2}\right\}\right]^{\frac{1}{2}} .
\end{aligned}
$$

In Eq. (4.6) the function $h\left(q^{2}\right)$ contains the hadronic structure and $V_{\mathrm{KM}}^{2}$ denotes the quark mixing angles, in this case $V_{\mathrm{KM}}^{2}=V_{u d}^{2}$. Here we shall not write down explicitly both factors [26].

At the end point of the spectrum,

$$
m_{\nu_{\tau}}=m_{\tau}-m_{\mathrm{had}}
$$

where $m_{\text {had }}=\left(q^{2}\right)^{\frac{1}{2}}$. Assuming that the $\nu_{3}$ is heavier than $\nu_{1,2}$ we get, instead of Eq. (4.6),

$$
\frac{d \Gamma}{d q^{2}}=\frac{G^{2} V_{\mathrm{KM}}^{2}}{8 \pi m_{\tau}^{3}}\left[\left(\left|V_{\tau 1}\right|^{2}+\left|V_{\tau 2}\right|^{2}\right) F_{0}+\left|V_{\tau 3}\right|^{2} F_{3}\right] h\left(q^{2}\right),
$$

where

$$
F_{0}=w\left(q^{2}, m_{\tau}^{2}, 0\right) \lambda^{\frac{1}{2}}\left(m_{\tau}^{2}, q^{2}, 0\right)
$$

and

$$
F_{3}=w\left(q^{2}, m_{\tau}^{2}, m_{\nu_{3}}^{2}\right) \lambda^{\frac{1}{2}}\left(m_{\tau}^{2}, q^{2}, m_{\nu_{3}}^{2}\right)
$$

Each part of the phase space functions $F_{0}, F_{3}$ have a different end point.

The second end point related to the massive contribution $m_{\nu_{3}}$ disappears when both massive and massless contributions are summed up, remaining only a shoulder in the distribution and the end point is related with the (almost) massless neutrinos. This is the end point observed experimentally.

Using $m_{\nu_{3}}=165,225 \mathrm{MeV}$ and typical values for the respective mixing angles in Eq. (4.10) we obtain Fig. 2. We see that the $m_{\nu_{3}}$ values given in (4.2) are allowed by the experimental data of the $\tau \rightarrow 5 \pi \nu$ decay. We also show in Fig. 2 a blowup of the region near the end point to emphasize the fact that all the three curves for $m_{\nu_{3}}=0,165,225 \mathrm{MeV}$ would be compatible with the three events found by ARGUS in this end point area $[5,10]$.

We have only studied the phase space contributions and have not taken into account the hadronic effects contained in $h\left(q^{2}\right)$. This function hides our ignorance on hadronic structure and it will, in general, modify the shape of the distribution but not affect the end point. However close to the end point the shape of the distribu- 


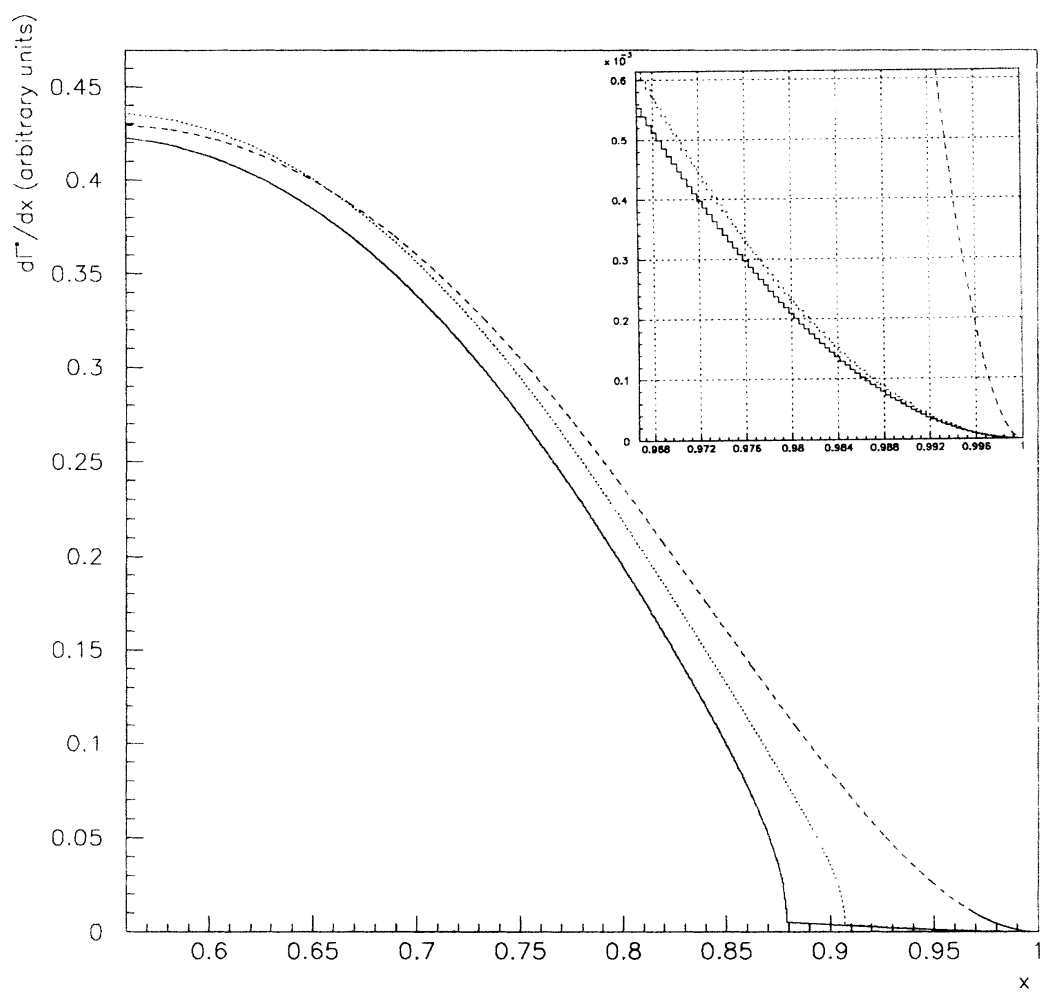

FIG. 2. Spectrum for $m_{\nu_{3}}=0$ (dashed line), $m_{\nu_{3}}=165 \mathrm{MeV}$ (dotted line), and $m_{\nu_{3}}=215 \mathrm{MeV}$ (continuous line). Here $d \Gamma^{*} / d x=\left(8 \pi m_{\tau}^{3} / G^{2} V_{\mathrm{KM}}\right)^{-1} d \Gamma / d x$, $G^{2}=G_{\mu}^{2} /\left(s_{\beta}^{2} s_{\gamma}^{2}+c_{\gamma}^{2}\right) c_{\beta}^{2}$, and $x=m_{h} / m_{\tau}$. The coefficient $\left(s_{\beta}^{2} s_{\gamma}^{2}+c_{\gamma}^{2}\right) c_{\beta}^{2}$ has been absorbed in the definition of $\Gamma$. We also shown the blowup of region near of end point.

tion does not depend very much on the resonant structure. On the other hand, the population of the spectrum in this region will be strongly modified [26]. For this reason, to be able to detect an effect of mixing it is necessary to study the population of the spectrum close to the end point.

\section{OTHER CONSTRAINTS}

\section{A. Neutrino oscillations}

The case of three light neutrino oscillations was consider in Ref. [27]. Here we will study the case of only two light neutrinos and a heavy one as in Ref. [18]. Let us start by summarizing at this stage our results for the mixing angles:

$$
\left(\begin{array}{ccc}
0.977 c_{\theta} & 0.977 s_{\theta} & 0.211 \\
-0.998 s_{\theta}-0.016 c_{\theta} & 0.998 c_{\theta}-0.016 s_{\theta} & 0.069 \\
0.071 s_{\theta}-0.2 c_{\theta} & -0.071 c_{\theta}-0.2 s_{\theta} & 0.975
\end{array}\right)
$$

The matrix above is orthogonal independently of the $\theta$.

As we said before, neutrinos produced in weak processes are linear combinations of the mass eigenstates $\nu_{l}=\sum_{i} V_{l i} \nu_{i}$ with $V_{l i}$ given in (2.2) and in particular in (5.1). Here we will write down the probability of finding a neutrino $\nu_{e}, \nu_{\mu}$ after a path length $R$ if at the origin it was a $\nu_{e}$, that is,

$$
\begin{aligned}
P\left(\nu_{e} \rightarrow \nu_{e}\right)= & \left|\left\langle\nu_{e}(L) \mid \nu_{e}(0)\right\rangle\right|^{2} \\
= & 1-2 c_{\beta}^{2} s_{\beta}^{2}\left(1-\cos \frac{2 \pi R}{L}\right) \\
& -2 c_{\theta}^{2} s_{\theta}^{2} c_{\beta}^{4}\left(1-\cos \frac{2 \pi R}{L_{12}}\right)
\end{aligned}
$$

and

$$
\begin{aligned}
P\left(\nu_{e} \rightarrow \nu_{\mu}\right)= & \left|\left\langle\nu_{\mu}(L) \mid \nu_{e}(0)\right\rangle\right|^{2} \\
= & h_{1}(\beta, \gamma, \theta)-h_{2}(\beta, \gamma, \theta) \cos \frac{2 \pi R}{L_{12}} \\
& -h_{3}(\beta, \gamma, \theta) \cos \frac{2 \pi R}{L}
\end{aligned}
$$

where we have defined

$$
\begin{gathered}
h_{1}(\beta, \gamma, \theta)=2 c_{\beta}^{2}\left[c_{\theta}^{2} s_{\theta}^{2} c_{\gamma}^{2}+\left(1-c_{\theta}^{2} s_{\theta}^{2}\right) s_{\gamma}^{2} s_{\beta}^{2}\right. \\
\left.+c_{\gamma}^{2} s_{\gamma}^{2} s_{\beta}^{2} c_{\theta}^{2} s_{\theta}^{2}\left(c_{\theta}^{2}-s_{\theta}^{2}\right)\right], \\
h_{2}(\beta, \gamma, \theta)=2\left[c_{\theta} s_{\theta}\left(c_{\gamma}^{2}-s_{\gamma}^{2} s_{\beta}^{2}\right)\right. \\
\left.+c_{\gamma} s_{\gamma} s_{\beta}\left(c_{\theta}^{2}-s_{\theta}^{2}\right)\right] c_{\theta} s_{\theta} c_{\beta}^{2}, \\
h_{3}(\beta, \gamma, \theta)=2 s_{\gamma}^{2} s_{\beta}^{2} c_{\beta}^{2} .
\end{gathered}
$$

As in Ref. [18], for the mass range we are considering here, neutrino oscillations occur with essentially two wavelengths. We have defined, in (5.2) and (5.3), 


$$
L_{i j}=\frac{2 \pi}{E_{i}-E_{j}}
$$

that is,

$$
L_{12}=\frac{4 \pi p}{\delta m^{2}}=2.5 \frac{p / \mathrm{MeV}}{\delta m^{2} /(\mathrm{eV})^{2}} \mathrm{~m}
$$

with

$$
\delta m^{2}=m_{\nu_{2}}^{2}-m_{\nu_{1}}^{2}
$$

and

$$
L_{13}=L_{23}=L=\frac{2 \pi}{m_{\nu_{3}}}=\frac{1.24}{m_{\nu_{3}} / \mathrm{MeV}} \times 10^{-12} \mathrm{~m} .
$$

The masses $m_{\nu_{1}}, m_{\nu_{2}}$, and of course $\delta m^{2}$, are still undetermined. Let us take, for instance, $\delta \mathrm{m}^{2}$ of the order of a few $\mathrm{eV}^{2}$. Of course for smaller $\delta m^{2}$ values the cosine involving $L_{12}$ in Eq. (5.2) must be taken into account. The short component has an oscillation length of the order of $10^{-15} \mathrm{~m}$. A more detailed study concerning neutrino oscillation data will be published elsewhere.

Let us consider the solar neutrino data. In this case $R \sim 10^{11} \mathrm{~m}$ and $L_{12} \approx 0.25 \mathrm{~m}$ if $\delta m^{2} \approx 1(\mathrm{eV})^{2}$. With this condition the oscillation has averaged out and one obtains

$$
P\left(\nu_{e} \rightarrow \nu_{e}\right)=1-2 c_{\beta}^{2} s_{\beta}^{2}-2 c_{\theta}^{2} s_{\theta}^{2} c_{\beta}^{4}
$$

Using the values in (5.1) and the experimental data given in Table I for $P\left(\nu_{e} \rightarrow \nu_{e}\right)$ [28] we obtain the allowed regions showed in Fig. 3. In Fig. 4 we show values for the angles compatible with those in Fig. 1. Note that with these angles the result of Davis, a suppression of about $27 \%$ of the solar standard model prediction, is not fitted at the $1 \sigma$ level. The lowest value of Eq. (5.4) is about $30 \%$.

Neutrino oscillations are only sensitive to small mass differences and cannot be used to detect mixing of heavy neutrinos directly. Notwithstanding, we see from (5.4) that even in the averaged situation the effect of the mixing with the heavy neutrino survives via the angle $\beta$.

The range for the $\theta$ angle compatible with the allowed area showed in Fig. 1, coming from Kamiokande II, Gallex, and SAGE data is

$$
\begin{aligned}
& 0.253<s_{\theta}^{2}<0.299 \\
& 0.701<s_{\theta}^{2}<0.747
\end{aligned}
$$

Hence, the mixing matrix in the leptonic sector which is consistent with all experimental data considered by us, for $m_{\nu_{3}}=165 \mathrm{MeV}$, is $\left(\begin{array}{ccc}0.817-0.848 & 0.490-0.536 & 0.200-0.222 \\ -(0.502-0.560) & 0.828-0.865 & <0.069 \\ -(0.192-0.129) & -(0.101-0.183) & 0.973-0.980\end{array}\right)$

Two final remarks. First, if we take into account the cosine term in Eq. (5.2) it is also possible to fit the Homestake data within our scheme. For example with $s_{\beta}^{2}=0.045, s_{\theta}^{2}=0.275$, and $L_{12} / R=2.5$ we obtain $P\left(\nu_{e} \rightarrow \nu_{e}\right)=0.26$. A more complete analysis should take into account the energy dependence of the neutrino spectra which contribute to each experiment.

A second remark concerns the "atmospheric neutrino problem" [29]. This consists in a suppression of the muon neutrino flux with respect to the electron neutrino one as observed by two experiments [30,31].

Usually in the two generation mixing approach the solution of both, the atmospheric and solar neutrino problems, require a fine-tuning of the parameters involved [15]. Preliminary calculations point out that this is also the case in the present situation. However it would be interesting to examine the Mikheyev-SmirnovWolfenstein (MSW) [32] effect in the context of this work. We are currently carrying this out.

\section{B. Cosmological constraints}

From cosmological constraints, stable neutrinos must have masses less than $40 \mathrm{eV}$ or greater than $2 \mathrm{GeV}$ [33]. In our scenario, of course, by construction neutrinos are not stable.

Earlier works about the upper bounds on the lifetime of a massive neutrino assumed $V-A$ interactions in annihilation processes of the massive neutrinos and that the principal decay mode is $\nu_{3} \rightarrow \nu_{e} \gamma[34,35]$. However, for a neutrino mass $m_{\nu_{3}} \gg m_{e, \mu}$ this decay [and the not Glashow-Iliopoulos-Maiani (GIM) suppressed $\nu_{3} \rightarrow$ $\nu_{e} \gamma \gamma$ ] are not dominant [35].

Note that as there are no changing flavor neutral currents at tree level, constraints as those in Ref. [36] do not apply to our case.

Here we will assume the mixing angles given in (5.6) and evaluate the lifetime for a heavy neutrino. In the mass range we are considering for the heaviest neutrino, 155-225 MeV, the main decays are $\nu_{3} \rightarrow l^{+} l^{-} \nu_{1,2}, l=$ $e, \mu$. The lifetime of heavy neutrinos in our context was studied by Kolb and Goldman [16]. Here we will treat again these decays. The partial widths are

TABLE I. Results of solar neutrino experiments. The flux is given as a function of the solar standard model prediction [49].

\begin{tabular}{llll}
\hline \hline Experiment & Process & $E_{\text {threshold }}(\mathrm{MeV})$ & Expt/SSM \\
\hline Davis et al. & $\nu_{e}+{ }^{37} \mathrm{Cl} \rightarrow e+{ }^{37} \mathrm{Ar}$ & 0.81 & $0.27 \pm 0.04$ \\
Kamiokande II & $\nu+e \rightarrow \nu+e$ & 7.5 & $0.49 \pm 0.05 \pm 0.06$ \\
SAGE & $\nu_{e}+{ }^{71} \mathrm{Ga} \rightarrow e+{ }^{71} \mathrm{Ge}$ & 0.24 & $0.44_{-0.18}^{+0.13} \pm 0.11$ \\
Gallex & $\nu_{e}+{ }^{71} \mathrm{Ga} \rightarrow e+{ }^{71} \mathrm{Ge}$ & 0.24 & $0.66 \pm 0.11 \pm 0.05$ \\
\hline \hline
\end{tabular}




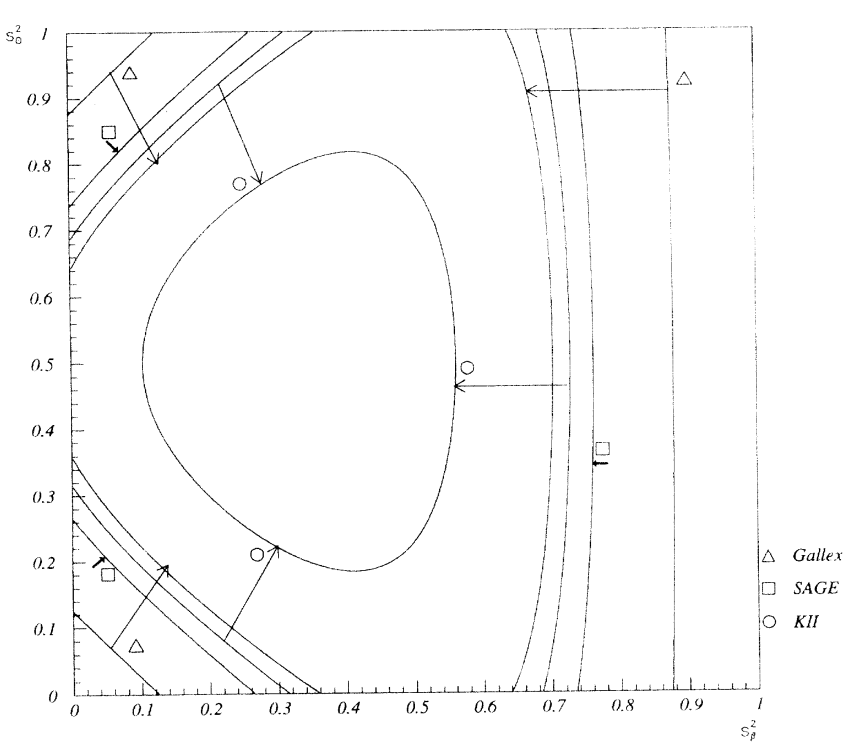

FIG. 3. Contour plot of Eq. (5.4) for SAGE, Gallex, and Kamiokande II using the values on Table I (within $1 \sigma$ ). The arrows shown the allowed contours for each experiment.

$$
\begin{aligned}
\Gamma\left(\nu_{3} \rightarrow l l \nu\right)= & \frac{G_{\mu}^{2} m_{\nu_{3}}^{5}}{192 \pi^{3}\left(1-\left|V_{\mu 3}\right|^{2}\right)\left(1-\left|V_{e 3}\right|^{2}\right)} \\
& \times\left[\left|V_{l 3}\right|^{2}\left(1-\left|V_{l 3}\right|^{2}\right) F\left(m_{l}, m_{\nu_{3}}\right)\right],
\end{aligned}
$$

and a similar expression for $\nu_{3} \rightarrow \mu e \nu$.

Notice that we have taken into account the appropriate modification of the $G_{\mu}$ constant given in Eq. (2.13). In fact, we leave in (5.7) all factors in such a way that it is

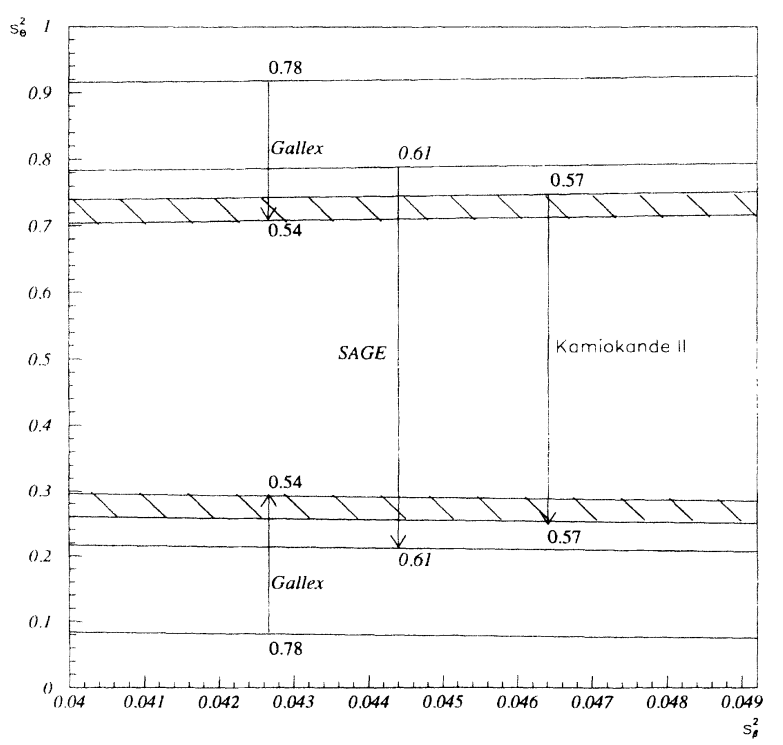

FIG. 4. Contour plot for Gallex, Kamiokande II, and SAGE data in the restricted region showed in black on Fig. 1. The shaded areas are the allowed regions for these experiments. clear that some cancellation of matrix elements occurs, otherwise we would obtained only the factor $\left|V_{e 3}\right|^{2}(1-$ $\left.\left|V_{e .3}\right|^{2}\right)$ for the case of electron decay and $\left|V_{\mu 3}\right|^{2}\left(1-\left|V_{\mu 3}\right|^{2}\right)$ for $\mu$ decay as in Ref. [16].

The function $F\left(m_{l}, m_{\nu_{3}}\right)$ is defined as

$$
\begin{aligned}
F\left(m_{l}, m_{\nu_{3}}\right)= & 2 \int_{y_{m}}^{y_{M}} \frac{\left(y^{2}-B^{\prime}\right)}{\left(k^{\prime}-y\right)^{3}}\left(k^{\prime}-y-\omega_{l}^{2}\right)^{2} \\
& \times\left\{\left(k^{\prime}-y-\omega_{l}^{2}\right) y\left(k^{\prime}-y\right)\right. \\
& +\left[\left(k^{\prime}-y\right)^{2}+\omega_{l}^{2}\left(k^{\prime}-y\right)\right. \\
& \left.\left.-2 \omega_{l}^{4}\right]\left(2 k^{\prime} y-y^{2}-B^{\prime}\right)\right\} d y
\end{aligned}
$$

where

$$
\begin{gathered}
k^{\prime}=1+\omega_{l}^{2}, \quad B^{\prime}=4\left(k^{\prime}-1\right), \quad \omega_{l}=\frac{m_{l}}{m_{\nu_{3}}}, \\
y_{m}=2 \omega_{l}, \quad y_{M}=1 .
\end{gathered}
$$

Here we will consider only the $\nu_{3} \rightarrow$ eev decay. The respective lifetime scaled from muon decay is

$$
\begin{aligned}
\tau\left(\nu_{3} \rightarrow e e \nu\right) & =\tau_{\mu}\left(\frac{m_{\mu}}{m_{\nu_{3}}}\right)^{5} \frac{1-\left|V_{\mu 3}\right|^{2}}{\left|V_{e 3}\right|^{2}} \frac{\Gamma_{00}^{\mu e}}{F\left(m_{e}, m_{\nu_{3}}\right)} \\
& =\tau_{\mu}\left(\frac{m_{\mu}}{m_{\nu_{3}}}\right)^{5} \frac{1-s_{\gamma}^{2} c_{\beta}^{2}}{s_{\beta}^{2}} \frac{\Gamma_{00}^{\mu e}}{F\left(m_{e}, m_{\nu_{3}}\right)},
\end{aligned}
$$

where $\tau_{\mu}$ is the muon lifetime. From (5.9) and the angles in (5.6) we see that the lifetime for the decay of $\nu_{3}$ into electrons is of the order of $10^{-6}-10^{-5} \mathrm{~s}$.

For the range (4.2) the decays $\nu_{3} \rightarrow \pi^{+} e^{-}$and $\nu_{3} \rightarrow$ $\mu^{+} e^{-} \nu$ are possible, they will not be considered here. Our purpose is only to indicate that there is no strong constraints coming from processes with cosmological consequences.

There are experiments which tried to observe neutrino decays $[37,38]$, however those experiments are sensitive to lifetimes of the order of $10^{-2}-10^{2} \mathrm{~s}$. Hence, their data are not directly applicable to our case.

For instance, in [37] it was assumed that the $\nu_{\tau}$ couples mainly to a single mass eigenstate, then an upper limit of the square of the matrix element $\left|V_{e i}\right|$ was obtained. In our case it is necessary to consider at least two of such matrix elements at once.

On the other hand, we must recall the following. In [37] the expected neutrino flux depends on the number of $D_{s}\left(N_{D_{s}}\right)$ and of $D$ mesons $\left(N_{D}\right)$ produced by protons in the dump. In computing this number it was assumed the following values for the branching ratio for the semileptonic decays $B\left(D \rightarrow \mu \nu_{3}\right)=0.1$ and $B\left(D_{s} \rightarrow \mu \nu_{3}\right)=0.03$. Notwithstanding, at present these branching ratios are $B\left(D \rightarrow \mu \nu_{3}\right) \leq 7.2 \times 10^{-4}[6]$ and $B\left(D_{s} \rightarrow \mu \nu_{3}\right)=\left(4.0_{-1.4-0.6}^{+1.8+0.8} \pm 1.8\right) \times 10^{-3}$ [39]. It means that the neutrino flux is reduced by a factor of a hundred with respect to the values used by the CHARM exper- 
iment [37], and that in fact higher values for the upper bounds on the mixing angles are allowed. On the other hand, in Ref. [38] the neutrino flux was calculated using Monte Carlo programs and we do not know if in this case the same branching ratios for the decays of the $D$ mesons were used.

In addition, we stress that in our case the lifetime of the heavy neutrino is such that it would decay before the detector.

\section{Astrophysical constraints}

Here we will discuss, briefly, the possible effects of our scenario in the solar neutrino flux and in the Supernova $1987 \mathrm{~A}$ data. In fact for neutrinos with mass $1.1 \mathrm{MeV}<m<14 \mathrm{MeV}$ the experimental detection of solar neutrinos have been considered in Ref. [40]. Neutrinos coming from the Sun, generated through the decay ${ }^{8} \mathrm{~B} \rightarrow{ }^{8} \mathrm{~B}_{e} e^{+} \nu$ must have a mass up to near $14 \mathrm{MeV}$ which is the threshold energy for this reaction. Those coming from $p p$ reaction have $m<1.44 \mathrm{MeV}$. Of course, a heavy neutrino would decay in flight producing $\nu_{e, \mu}$ and charged leptons.

The observation of $\bar{\nu}_{e}$ 's coming from the Supernova 1987A implies a constraint on their lifetime:

$$
\tau_{\nu_{e}} / m_{\nu_{e}} \gtrsim 6 \times 10^{5} \mathrm{~s} / \mathrm{eV} \text {. }
$$

However this constraint is valid for neutrinos reaching the Earth's orbit, and no mixing in the leptonic sector. In fact, we have seen in Sec. V B that for a mass of 165 $\mathrm{MeV}$ the mixing angles are such that the lifetime of the heavy neutrino is of the order of $10^{-6}-10^{-5} \mathrm{~s}$. It means that this neutrino can appear only through the mixing effect as it is kinematically forbidden at the characteristic energies of the Supernova and must decay before reaching the Earth's orbit. We see that only the lightest neutrinos $\nu_{1,2}$ are constrained from these data.

On the other hand, since neutrinos are massive Dirac particles the right-handed components can be produced in the hot Supernova via helicity flip. This produce an extra source of cooling in the Supernova since this righthanded neutrinos are almost sterile. The relevant spinflip processes are $N N \rightarrow N N \bar{\nu}_{R} \nu_{R}$ (bremsstrahlung), $N \nu_{L} \rightarrow N \nu_{R}$ and $e^{+} e^{-} \rightarrow \bar{\nu}_{R} \nu_{R}$. However bounds coming from this processes in Supernova are valid only for small enough neutrino masses and do not apply to our heavy neutrino. It is not thermally emitted from the Supernova $[41,42]$.

\section{CONCLUSIONS}

We have examined some weak processes which are modified by a three generation mixing in the leptonic sec- tor and found compatibility regions for $m_{\nu_{3}}$ and mixing angles. These parameters are likely to have consequences elsewhere.

The main result of this paper is that the current upper limit on the $\tau$-neutrino mass " $m_{\nu_{\tau}}$ " does not seem to exclude a heavy neutrino $155 \mathrm{MeV}<m_{\nu_{3}}<225 \mathrm{MeV}$ mixed up with the light ones, and solving at the same time a possible deviation of the $\tau-\mu$ universality. However, our analysis will still be valid if no deviation from $\tau-\mu$ universality is confirmed by data. Only the value for $m_{\nu_{3}}$ and mixing angles will be different. If the new data imply lowest values for the $\nu_{3}$ mass and, as in $\nu_{3}$ decays photons are ultimately produced [43] it will be interesting to study astrophysical bounds more in detail.

Of course, there are other experiments we could have considered as the $\nu_{\mu} e \rightarrow \nu_{\mu} e$ cross section [12], measurements of the Michel parameter in muon decay [44], etc., but we do not expect they to produce strongest constraints than the ones we have analyzed.

The $\mu-e$ universality has been also verified in the kaon decays [45]. In fact, in the mass range (4.2) the decays $K \rightarrow e(\mu) \nu_{i}$ with $i=1,2,3$ occur. In particular, the branching ratio of the kaon decays, $\Gamma(K \rightarrow e \nu) / \Gamma(K \rightarrow$ $\mu \nu)$ have been measured [46] and, in principle, provide another confirmation of the $e \mu$ universality, but in this case the structure-dependent radiative correction is expected to be $\sim 1000$ times larger than that for the pion decays partial widths [3]. For this reason we will not analyze these decays in this work. However we recall that in the literature a similar expression to (3.1) was also assumed.

Notice that in the extension of the standard model we are considering there are no flavor changing neutral currents at tree level, since they are GIM suppressed [47], and for this reason it is not necessary to consider processes producing $\mu e$ events.

We recall that the final allowed values for masses and mixing angles must be obtained taken into account radiative corrections [48]. Any way, radiative corrections imply a reduction of about $\sim 4 \%[3]$ and will not modify qualitatively our results.

\section{ACKNOWLEDGMENTS}

We thank Fundação de Amparo à Pesquisa do Estado de São Paulo (FAPESP) (O.L.G.P.) for full financial support and Conselho Nacional de Desenvolvimento Científico e Tecnológico (CNPq) (V.P.) for partial and full (R.Z.F.) financial support. We are very grateful to C.O. Escobar for stimulating discussions at the early stage of this work. We also thank M.M. Guzzo for very useful comments concerning solar neutrino data.
[1] C.O. Escobar, O.L.G. Peres, V. Pleitez, and R. Zukanovich Funchal, Europhys. Lett. 21, 169 (1993).

[2] A. Pich, in Heavy Flavors, edited by A. J. Buras and M. Lindler (World Scientific, Singapore, 1991).

[3] For a recent review on this subject, see D.A. Bryman, Comments Nucl. Part. Phys. 21(2), 101 (1993); T. Nu- mao, Mod. Phys. Lett. A 7, 3357 (1992).

[4] BES Collaboration, J.Z. Bai et al., Phys. Rev. Lett. 69, 3021 (1992).

[5] ARGUS Collaboration, H. Albrecht et al., Phys. Lett. B 202, 211 (1992).

[6] Particle Data Group, K. Hikasa et al., Phys. Rev. D 45, 
S1 (1992).

[7] D.I. Britton et al., Phys. Rev. Lett. 68, 3000 (1992).

[8] S. Weinberg, Phys. Rev. Lett. 19, 1264 (1967); A. Salam, in Elementary Particle Theory: Relativistic Groups and Analyticity (Nobel Symposium No. 8), edited by N. Svartholm (Almqvist and Wiksell, Stockholm, 1969), p. 367; S. Glashow, Nucl. Phys. 22, 579 (1972).

[9] M. Kobayashi and K. Maskawa, Prog. Theor. Phys. 49, 652 (1973).

[10] ARGUS Collaboration, H. Albrecht et al., Phys. Lett. B 292, 221 (1992).

[11] J.D. Bjorken and C.H. Llewellyn Smith, Phys. Rev. D 27, 887 (1973); C.H. Albright, ibid. 12, 1319 (1975); J.L. Rosner, Nucl. Phys. B126, 124 (1977); D. Rein, L.M. Sehgal, and P.M. Zerwas, ibid. B138, 85 (1978); C.N. Leung and J.L. Rosner, Phys. Rev. D 28, 2205 (1983).

[12] M. Gronau, Phys. Rev. D 28, 2762 (1983).

[13] M. Gronau, C.N. Leung, and J.L. Rosner, Phys. Rev. D 29, 2539 (1984).

[14] L.S. Littenberg and R.E. Shrock, Phys. Rev. Lett. 68, 443 (1992).

[15] C. Rubbia, "The renaissance of experimental neutrino physics," Report No. CERN-PPE/93-08, 1993 (unpublished).

[16] E.W. Kolb and T. Goldman, Phys. Rev. Lett. 43, 897 (1979).

[17] R. Shrock, Phys. Lett. 96B, 159 (1980); Phys. Rev. D 24, 1232 (1981); 24, 1275 (1981).

[18] J.N. Ng, Nucl. Phys. B191, 125 (1981); Phys. Lett. 99B, 53 (1981).

[19] P. Kalyniak and J.N. Ng, Phys. Rev. D 24, 1874 (1981).

[20] R.R.L Sharma and N.K. Sharma, Phys. Rev. D 29, 1533 (1984).

[21] L. Maiani, Phys. Lett. 62B, 183 (1976).

[22] R. Abela et al., Phys. Lett. 105B, 263 (1981); D.A. Bryman et al., Phys. Rev. Lett. 50, 1546 (1983); G. Bernardi et al., Phys. Lett. 166B, 479 (1986); N. de Leener-Rosier et al., Phys. Lett. B 177, 228 (1986); G. Azuelos et al., Phys. Rev. Lett. 56, 2241 (1986).

[23] D.I. Britton et al., Phys. Rev. D 46, R885 (1992).

[24] For this notation see the first reference in Ref. [17].

[25] W.F.L. Hollik, Fortschr. Phys. 38(3), 165 (1990).

[26] J.J. Gomez-Cadenas et al., Phys. Rev. D 41, 2179 (1990).

[27] V. Barger, K. Whisnant, and R.J.N. Phillips, Phys. Rev. D 22, 1636 (1980), and more recently in D. Harley, T.K. Kuo, and J. Pantaleone, ibid. 47, 4059 (1993).

[28] Homestake Collaboration, R. Davis et al., in Proceedings of the XXI International Cosmic Ray Conference, Adelaide, Australia, 1989, edited by R.J. Protheroe (University of Adelaide Press, Adelaide, 1990), Vol. 12, p. 143; SAGE Collaboration, A. I. Abazov et al., Phys. Rev. Lett. 67, 3332 (1991); A. Gavrin, in Proceedings of the 26th International Conference on High Energy Physics, Dallas, Texas, 1992, edited by J. Sanford, AIP Conf. Proc. No. 272 (AIP, New York, 1993); Kamiokande II
Collaboration, K.S. Hirata et al., Phys. Rev. D 44, 2241 (1991); T. Kajita, in Proceedings of the 26th International Conference on High Energy Physics, this reference; GALLEX Collaboration, P. Anselmann et al., Phys. Lett. B 285, 390 (1992); D. Vignaud, in Proceedings of the 26th International Conference on High Energy Physics, this reference, presented by $\mathrm{F}$. von Feilitzsch, in Proceedings of the 26th International Conference on "Neutral Currents Twenty Years Later," Paris, France, 1993 (to be published).

[29] For a detailed analysis see, for instance, G.L. Fogli, E. Lisi, and D. Montanino, Phys. Rev. D 49, 3626 (1994).

[30] Kamiokande Collaboration, K.S. Hirata et al., Phys. Lett. B 280, 146 (1992); IMB Collaboration, D. Casper et al., Phys. Rev. Lett. 66, 2561 (1991); IMB Collaboration, R. Becker-Szendy et al., Phys. Rev. D 46, 3720 (1992).

[31] However we must recall that there are two other experiments that do not observe this effect. See, Fréjus Collaboration, Ch. Berger et al., Phys. Lett. B 227, 489 (1989); 245, 305 (1990); NUSEX Collaboration, M. Aglietta et al., Europhys. Lett. 8, 611 (1989).

[32] L. Wolfenstein, Phys. Rev. D 17, 2369 (1979); S.P. Mikhayev and A.Yu. Smirnov, Yad. Fiz. 42, 1441 (1985) [Sov. J. Nucl. Phys. 42, 913 (1985)].

[33] B.W. Lee and S. Weinberg, Phys. Rev. Lett. 39, 165 (1977).

[34] D.A. Dicus, E.W. Kolb, and V.L. Teplitz, Phys. Rev. Lett. 39, 168 (1977); D.A. Dicus, E.W. Kolb, V.L. Teplitz, and R.V. Wagoner, Phys. Rev. D 17, 1529 (1978).

[35] S. Sarkar and A.M. Cooper, Phys. Lett. 148B, 347 (1984).

[36] A.A. Natale, Phys. Lett. 141B, 323 (1984).

[37] CHARM Collaboration, F. Bergsma et al., Phys. Lett. 128B, 361 (1983).

[38] G. Bernardi et al., Phys. Lett. 166B, 479 (1986).

[39] WA75 Collaboration, S. Aoki et al., Prog. Theor. Phys. 89, 131 (1993).

[40] D. Toussaint and F. Wilczek, Nature (London) 289, 777 (1981).

[41] J.A. Grifols, invited talk at Escuela Latinoamericana de Física, La Plata, Argentina, 1993 (unpublished).

[42] G.G. Raffelt, Mod. Phys. Lett. 5, 2581 (1990).

[43] A.D. Dolgov and Ya. B. Zeldovich, Rev. Mod. Phys. 53, 1 (1991).

[44] M.S. Dixit, P. Kalyniak, and J.N. Ng, Phys. Rev. D 27, 2216 (1983).

[45] K.S. Heard et al., Phys. Lett. 55B, 327 (1975).

[46] J. Heintze et al., Phys. Lett 60B, 302 (1976); R.S. Hayano et al., Phys. Rev. Lett. 49, 1305 (1982).

[47] S.L. Glashow, J. Iliopoulos, and L. Maiani, Phys. Rev. D 2, 1285 (1970).

[48] P. Kalyniak and J.N. Ng, Phys. Rev. D 25, 1305 (1982).

[49] See Harley et al., [27], and references therein. 